\title{
EL TRABAJO DE LABORATORIO EN CURSOS DE FÍSICA DESDE LA TEORÍA DE CAMPOS CONCEPTUALES
}

\section{Laboratory work in physics in the light of the conceptual fields' theory}

\author{
Ma. MaiteAndrés Z. ${ }^{1}$ \\ Marta A. Pesa ${ }^{2}$ \\ Marco Antonio Moreira ${ }^{3}$
}

\begin{abstract}
Resumen: Los Trabajos de Laboratorio, dirigidos a la resolución de problemas de ciencia, implican el abordaje de situaciones problema mediante tareas y subtareas propias del quehacer científico. El proceso de aprendizaje durante el desarrollo de estos trabajos se analiza desde la Teoría de Campos Conceptuales propuesta por Gérard Vergnaud. Esta teoría considera el saber y el saber hacer como dos aspectos indivisibles en el desarrollo conceptual; y, permite analizar la relación entre conocimientos explícitos y conceptualizaciones implícitas a partir de las acciones de los sujetos en situaciones específicas. El desarrollo cognitivo se concibe en el marco de campos conceptuales, constituidos por conjuntos de: situaciones, conceptos y reglas de acción, y representaciones simbólicas, y centrado en la asimilación y acomodación de esquemas del sujeto en acción. Se presenta y discute un modelo (MAT$\mathrm{LaF}$ ) elaborado para la comprensión de los procesos cognitivos en un trabajo de laboratorio.
\end{abstract}

Palabras clave: conceptos-en-acción. teoremas-en-acción. trabajo de laboratorio. aprendizaje en el laboratorio de Física.

\begin{abstract}
This paper looks at laboratory work addressed to science problem solving which implies approaching problematic situations by means of tasks and subtask s typical of the scientific investigation. The learning process during the development of these tasks is analyzed from the perspective of the conœptual fields theory, proposed by $\mathrm{G}$ érard $\mathrm{V}$ ergnaud. This theory considers knowledge and knowing how to do as two indivisible aspects of conceptual development, and allows the analysis of the relationship between ex plicit knowledge and implicit oonceptions using students' actions in specific situations. Cognitive development as conceived in the framework of conceptual fields is made up of sets of situations, concepts and action rules, and symbolic representations, and focuses on assimilation schemes and accomodation of the subjects' action schemes. A model (MA TL aF) is presented, discussed and elaborated to understand the cognitive process in the laboratory work.
\end{abstract}

Keywords: C onœpts-in-action. theorems-in-action. laboratory work. learning in Physics laboratory.

\footnotetext{
${ }^{1}$ D epartamento de Matemática y Física, Universidad Pedagógica Ex xerimental Libertador, Instituto Pedagógico de Caracas. Caracas, V enezuela. E -mail: maitea@ cantv.net

${ }^{2}$ D epartamento de Física, Facultad de Ciencias E x actas y Tecnología, U niversidad N acional de Tucumán, T ucumán, A rgentina. E-mail: mpesa@ herrera.unt.edu.ar

${ }^{3}$ Instituto de Física, U niversidade do Rio G rande do Sul, Porto A legre, Brasil. E -mail: moreira@ if.ufrgs.br
} 


\section{Introducción}

El trabajo de laboratorio (TL) en los cursos de física puede tener diferentes modalidades e intenciones. Los autores hemos considerado el TL a partir de una situación problemática, en la cual los contenidos teóricos y experimentales asociados a ella, están en permanente relación e interdependencia, "tal como" ocurre en la actividad de investigación científica; en consecuencia, su resolución implica una compleja actividad cognitiva que requiere de diversos campos de conocimientos. Con el fin de analizar y orientar la investigación educativa en relación con los TL, hemos tomando un referencial cognitivo además del epistemológico, para proponer un modelo que representa la dinámica cognitiva de los estudiantes enfrentados a la resolución de situaciones problemáticas en el contexto de un laboratorio de Física básica. El modelo está basado en la Teoría de Campos Conceptuales propuesta por Vergnaud (1990).

\section{La Teoría de Campos Conceptuales}

La teoría de Campos Conceptuales (Vergnaud, 1990) constituye un marco referencial cognitivo para el estudio del desarrollo y aprendizaje de competencias complejas. Su potencialidad para la investigación sobre el aprendizaje de la Física radica en la posibilidad de comprender procesos que subyacen a la cognición, en particular, a la construcción de representaciones internas del sujeto. El mayor desarrollo de la teoría ha estado en el ámbito de la matemática, lo cual no la hace exclusiva de ese dominio. Se encuentran trabajos recientes en educación en Física con este marco referencial relacionados con: i) resolución de problemas y la interpretación de las dificultades en la construcción de modelos mentales ante enunciados de problemas novedosos (Escudero et al., 2003); ii) el estudio de los invariantes operatorios presentes en los esquemas y la efectividad de las representaciones simbólicas para la conceptualización en física (Weil-Barais e Vergnaud, 1990; Stipcich et al., 2004; Bravo e Pesa, 2004a; 2004b).

El referencial propuesto por Vergnaud parte del siguiente principio piagetiano: la actividad del sujeto constituye el eje central del aprendizaje y del desarrollo cognitivo, por ser ésta el factor más importante en el proceso de adaptación psicológica (Vergnaud, 1996). En este proceso de elaboración pragmática, el desarrollo cognitivo es moldeado por las acciones de los sujetos en situaciones concretas y por las conceptualizaciones subyacentes a ellas (Vergnaud, 1990). A diferencia de Piaget, Vergnaud toma como referencia el contenido del conocimiento y el análisis del dominio de ese conocimiento (Vergnaud, 1994; Moreira, 2002).

Entre los conceptos básicos de esta teoría se tiene el de Campo Conceptual (CC), el cual se concibe como "un onjunto informal y heterogéneo de problemas, situaciones, conœeptos, relaciones, estructuras, contenidos y operaciones de pensamiento, conectados unos a otros y, probablemente, entrelazados durante el proceso de adquisición" (Vergnaud, 1982). En otras palabras, un CC se constituye "como un conjunto de problemas y situaciones cuyo tratamiento requiere conceptos, procedimientos y representaciones de tipos diferentes, pero íntimamente relacionados" (Moreira, 2002).

Para Vergnaud (Moreira, 2002) la idea de concepto es central y lo considera constituido por tres conjuntos: i) las situaciones (referente), ii) los conceptos y teoremas-en-acción (significado), y iii) las representaciones simbólicas (lenguaje, gráficos, sentencias formales, diagramas,...) que 
son utilizadas para representar los conceptos, sus propiedades, las situaciones y los procedimientos de tratamiento, y constituyen el significante de los conceptos (Vergnaud, 1998; Rodríguez e Moreira, 2004).

Es importante destacar que se entiende por situación, a una tarea compleja (combinación de subtareas) cuya dificultad depende de la conceptualización para abordarla y no de la cantidad de subtareas; por otra parte, cada situación pone en acción algunas propiedades de los conceptos y de los teoremas asociados, dándole sentido a estos. D entro de cada CC, las situaciones se pueden agrupar en clases, en función de las propiedades de los conceptos que se requieren para su solución.

Los estudiantes van desarrollando sus conceptualizaciones (conocimientos) frente a las situaciones que van dominando, proceso éste lento y complejo con avances y retrocesos (Vergnaud, 1990). En el contexto de aprendizaje en el aula, se torna entonces fundamental identificar el contenido a aprender en términos de CC, y además, ver la enseñanza como un conjunto de situaciones a resolver por los estudiantes, clasificadas y jerarquizadas según su efectividad para el aprendizaje significativo.

D esde este referencial cabe preguntarse ¿cómo se abordan las situaciones? Una primera respuesta es que el comportamiento ante una situación dada está dirigido por esquemas. Un esquema es la organización invariante de la conducta para una clase de situaciones; en cada esquema encontramos elementos cognitivos (conocimientos-en-acción) que permiten actuar frente a una situación. Es entonces en los esquemas donde debemos centrar la investigación, indagar en ellos los conocimientos-en-acción, que tornan operatoria la acción de los estudiantes (Vergnaud, 1990; 1998; 1994).

Las situaciones son las que dan sentido a los conceptos. Pero, el sentido no está en las "situaciones en sí mismas", sino en la "relación del sujeto on las situaciones y con los significantes", es decir, con los esquemas (Moreira, 2002).

En general, los esquemas contienen varios ingredientes (Vergnaud, 1998):

- Invariantes operatorios. Son los conceptos-en-acción relevantes para la identificación y selección de la información, y los teoremas-en-acción, verdaderos o falsos, que relacionan los conceptos-en-acción, permitiéndole al individuo la producción de inferencias y la selección de reglas de acción para dominar la situación. Para la enseñanza son estos aspectos los que interesan investigar, ya que ellos contienen una parte explícita que se apoya en una conceptualización implícita, que no es ni verdadera ni falsa, tan sólo resulta ser operatoria (Vergnaud, 1990; 1998).

- M etas y anticipaciones. Estos permiten identificar las situaciones. En el aula de clase, deben ser debatidas y puestas en evidencia.

- Reglas de ación. Un esquema contiene reglas del tipo "si.... entonces" a partir de las cuales se genera una secuencia de acción específica para la situación; reglas de búsqueda de información y de control de resultados de acción.

- Posibilidades de inferencias. Razonamientos que permiten evaluar, "aquí y ahora", las reglas y anticipaciones a partir de los invariantes operatorios que dispone el sujeto. Se efectúan con los tres ingredientes anteriores durante la actividad frente a la situación.

Como se señaló, la teoría de CC concibe el proceso de conceptualización a partir de la interacción entre esquemas y situaciones. Además, considera indisoluble la relación entre: situaciones (referente que da sentido a los conceptos), invariantes operatorios (significado de 
los conceptos y fundamento de los esquemas) y, representaciones (lingǘsticas y no lingüísticas) de los conceptos, sus propiedades y los procedimientos para su aplicación (significante de los conceptos).

En el marco de la teoría de CC, las situaciones pueden ser conocidas o novedosas. Las primeras son identificadas por la persona como miembros de una clase ante las cuales dispone de competencias para abordarlas casi de manera inmediata, a veces con conductas bastante automatizadas, activadas por esquemas de asimilación específicos. El segundo tipo de situación, novedosas, no puede ser asociada con ninguna clase conocida, por lo que la persona no dispone de todas las competencias necesarias para abordarla, esto la lleva a un proceso de reflexión y exploración en el que se activan diferentes esquemas que serán acomodados, separados o combinados, propiciando la construcción de nuevos esquemas para nuevas situaciones. El funcionamiento cognitivo del sujeto ante una clase de situaciones nueva puede irse progresivamente automatizando, aunque siempre habrá un grado de decisión consciente que le permite identificar la particularidad de la situación (Vergnaud, 1990).

En el caso de situaciones novedosas, Moreira y Grecca (2002) plantean que al momento de enfrentarse a una situación nueva se forman representaciones (Modelos Mentales, $\mathrm{MM}$ ) en la memoria a corto plazo como modelos de trabajo para la solución de la tarea, estos se consideran mediadores entre la situación y los esquemas que se mantienen como estructuras en la memoria a largo plazo (conocimiento de la persona). D e este conocimiento se activan uno 0 más "tokens" que guían el proceso de elaboración y recursividad de los MM, en la medida en que se determinan los elementos que según el sujeto se aplican a la situación (conceptos-en-acción) y las propiedades que para el sujeto son verdaderas respecto a dicha situación (teoremas-en-acción), se van identificando, progresivamente, los posibles elementos de un nuevo esquema. Las inferencias y predicciones que se producen a lo largo del proceso de dar cuenta de la situación, están fuera de este esquema en construcción, se producen en los $\mathrm{MM}$ a nivel de las representaciones de la memoria a corto plazo. Por ello, se estima que en la familia de MM generados, se pueden diferenciar unos elementos empleados para abordar la situación y que luego son desechados, de otros que se repiten en la recursividad de los modelos, por lo que resultan más estables, equivalen a invariantes operatorios. Estos de resultar efectivos conformarán con otros IO provenientes de la memoria de largo plazo, el esquema en construcción. Es decir, el nuevo esquema estará conformado tanto por invariantes que ya integraban los esquemas que no funcionaban como por nuevos invariantes generados en el proceso recursivo de construcción de MM para dar cuenta de la situación nueva y sus variantes, o sea, una nueva clase de situaciones. A efecto del aprendizaje, la identificación de estos invariantes resulta relevante para guiar el desarrollo conceptual de los estudiantes, pues son precursores para la construcción de nuevos esquemas asociados a nuevas situaciones.

Por lo expuesto, este referencial teórico parece fructífero para investigar el proceso de aprendizaje en el contexto de resolución de problemas en el laboratorio.

\section{El trabajo de laboratorio: aprendizaje de la interrelación teoría-práctica}

El trabajo de laboratorio (TL) en la enseñanza es considerado, por muchos docentes, como una actividad de aprendizaje útil para una diversidad de funciones (Kerr,1963, apud 
Barberá \& Valdés, 1994; Lynch, 1987, apud Hodson, 1994; Hodson, 1994; Andrés, 2002). Sin embargo, en la práctica se observa un fuerte énfasis en el aprendizaje de destrezas y técnicas de recolección y procesamiento de datos experimentales, con poca o ninguna relación explícita con referentes teóricos o modelos; así como, una falta de claridad en cuanto a los objetivos de aprendizaje que se espera lograr con el trabajo de laboratorio. Esta falta de coherencia entre la finalidad asignada al laboratorio en términos de aprendizaje y lo que se hace en la práctica, tanto en el aula (Andrés, 2002) como en la investigación (Tobin et al., 1994), no ha permitido evaluar la efectividad del laboratorio en la enseñanza; en tal sentido, es primordial precisar el rol del TL en el contexto del aula de ciencias.

D iversos autores (Hodson, 1984; Gil et al., 1991; D uit, 1995; Barberá \& Valdés, 1996; Sére, 2002) consideran que el TL en la enseñanza de las ciencias es importante para: integrar lo conceptual y lo fenomenológico; establecer una conexión dialéctica entre datos y teoría; y sobre todo, promover el desarrollo de una visión de la naturaleza de la ciencia más cercana al quehacer científico.

En ciencia es difícil pensar en una actividad experimental, desligada de las ideas o componentes teóricos que representan el mundo físico. Sin embargo, la relación teoría - práctica puede ser vista desde diferentes posiciones epistemológicas (Sére, 2002; Andrés e Pesa, 2002), las cuales se reflejarán en la acción didáctica. Una de la formas que toma la acción didáctica, definida como: la práctica ayuda a aprender la teoría, es la dirigida a verificar o desaubrir relaciones teóricas 0 conceptos. G eneralmente, en estos casos aflora una perspectiva de ciencia que considera: el conocimiento como algo estable y verdadero; las leyes físicas como generalizaciones inductivas; y los modelos como réplicas de la realidad. D esde esta posición, lo metodológico es sólo necesario para producir el conocimiento científico, por ello en el laboratorio se enseñan técnicas independientes de los conocimientos teóricos.

O tra forma de traducir la relación teoría-práctica en el laboratorio es considerar que la teoría se emplea en la prádica, en el sentido de que el mundo de los objetos es inseparable del mundo de los modelos; en el contexto educativo, esto implica que las actividades experimentales requieren de los estudiantes un conocimiento previo en relación a la teoría, y una toma de conciencia en cuanto al rol que ésta tendrá en los diferentes momentos del trabajo de laboratorio (Sére, 2002).

D entro de este enfoque encontramos una propuesta didáctica que considera el TL como problemas abiertos en analogía a la investigación científica, en donde, la metodología científica se hace explícita y está ligada de manera indisoluble a los marcos conceptuales en los cuales se inserta el trabajo práctico, intentando producir cambio metodológico y cambio conceptual en los estudiantes, bajo la premisa de que la producción de conocimientos en la ciencia es equivalente al aprendizaje de la ciencia (Gil et al., 1991; Salinas, 1994; Gil e Valdés, 1996). Estos autores han elaborado secuencias didácticas ${ }^{4}$ que siguen la metodología científica, convirtiendo el laboratorio en un espacio de investigación científica, donde el profesor es el investigador experto que orienta a los estudiantes, investigadores noveles, que trabajan en un contexto de ciencia normal en el sentido khuniano.

\footnotetext{
${ }^{4}$ Secuencia de actividades científicas: discusión cualitativa, observación, proposición de conjeturas, elaboración de diseños, análisis de resultados, perspectivas, integración y comunicación.
} 
Sin embargo, consideramos que una debilidad de esta propuesta es que no toma en cuenta el proceso de aprendizaje y los obstáculos de orden cognitivo que confrontan los estudiantes al llevar a cabo cada una de las actividades de la secuencia; centrándose en aspectos epistemológicos y actitudinales. En muchos casos, los estudiantes tienen poca experiencia en la resolución de este tipo de situaciones desde la perspectiva de la ciencia; y su visión acenca de la naturaleza de la ciencia está marcada por una epistemología centrada en lo sensorial (D river et al., 1996). Por ello, al enfrentarlos a problemas abiertos se corre el riesgo de que fracasen por falta de conocimientos, y en consecuencia, el trabajo resulta sin significado y de manera mecánica. Tampoco parece existir una conexión epistemológica entre hacer ciencia y aprender ciencia, y por lo tanto, enseñar ciencia (Kirschner, 1992), como lo suponen los autores de la propuesta. Además, hay siempre que distinguir entre educación científica y entrenamiento científico. La escuela se debe ocupar de la educación científica. Todo ello, reafirma lo que plantea el grupo de investigación coordinado por Sére (2002), en cuanto a que la enseñanza del dominio metodológico inherente a los TL continúa siendo un problema de investigación.

En este trabajo, estamos de acuerdo con que en el laboratorio predomina el aprendizaje del dominio metodológico en interrelación indisoluble con algún marco teórico de referencia asociado a la situación planteada. Y que dentro de ese dominio, se identifican procesos típicos del quehacer de la ciencia, como: generar predicciones, formular hipótesis, seleccionar métodos, diseñar secuencias experimentales, recolectar, procesar, analizar e interpretar datos, elaborar síntesis y conclusiones, y derivar nuevas preguntas o acciones para seguir profundizando e investigando. Pero, esta interrelación entre teoría, modelos y experimento ocurre de manera no explícita y depende de cada situación; por lo que, con miras a facilitar la toma de

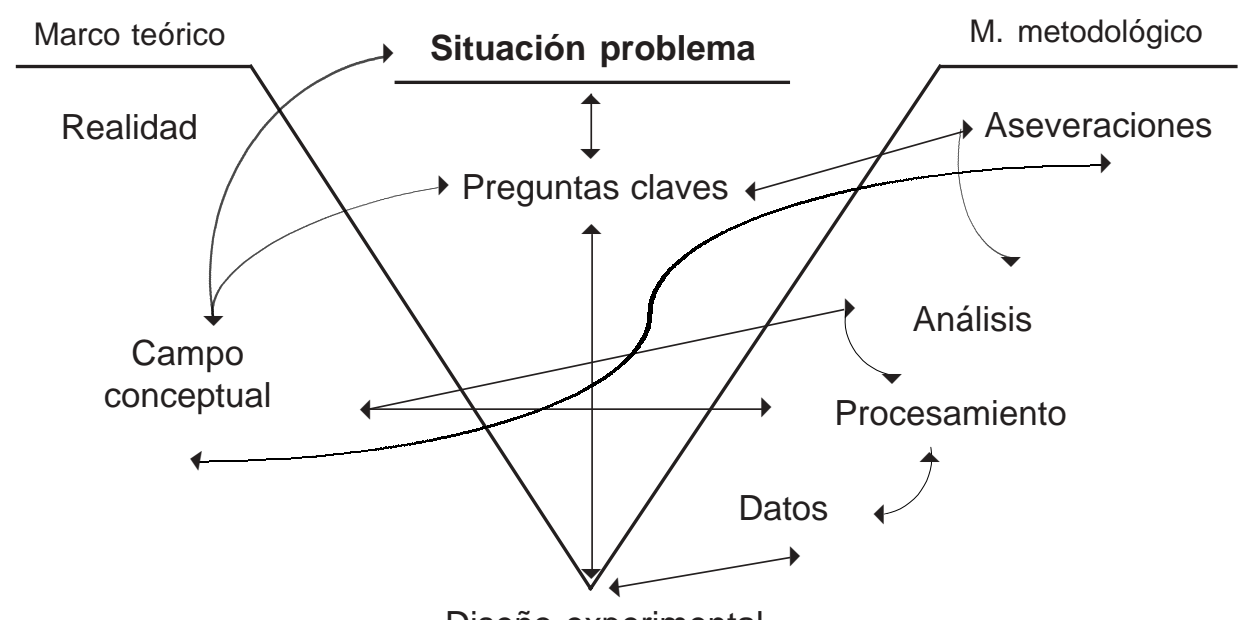

Diseño experimental

Figura 1. Dinámica del desarrollo de un trabajo de laboratorio a partir de una situación problema, visto desde el quehacer de la ciencia. 
conciencia de esta dinámica, la hemos representado en la $\mathrm{V}$ epistemológica descrita por $\mathrm{G}$ owin (Novak e G owin, 1984) (Figura 1).

Además, consideramos que estos procesos del dominio metodológico son una tarea compleja desde lo cognitivo, pues demandan conceptos y procedimientos metodológicos a la luz de algún referencial teórico para su resolución. Estos conocimientos deben ser aprendidos por los estudiantes, en consecuencia, deben ser declarados como metas explícitas de aprendizaje durante los TL.

Adicionalmente, resulta pertinente tomar en cuenta el desarrollo conceptual del estudiante en relación con el dominio teórico asociado a la situación del TL, a fin de identificar aspectos que también pudieran ser incluidos como objetivos de aprendizaje. También es posible que se requieran conocimientos de otros campos conceptuales demandados por procedimientos experimentales más generales propios del TL, como es el caso de los principios de funcionamiento inherentes a los equipos e instrumentos de medición.

Por otra parte, la actividad experimental en el aula parece ser un espacio importante para el desarrollo de una imagen de la ciencia (Leach, 1999; Leach et al., 2001). Para ello, es necesario que la comprensión de la naturaleza de la ciencia, su metodología y los mecanismos sociales e institucionales que operan en ella se planteen como objetivos de aprendizaje explícitos, como por ejemplo: i) reconocer que los modelos se basan en abstracciones (masa puntual, medios sin fricción, y otros), y que estos son construidos dentro de marcos teóricos que dan cuenta de eventos del mundo real; ii) reconocer las estrategias de indagación dentro de un campo disciplinar y establecer los procedimientos para implementarlas en una situación particular; iii) darse cuenta de lo que sería una apropiada explicación y argumentación; iv)analizar la información y datos que se produce en las investigaciones, así como, las técnicas más apropiadas para su recolección y análisis; v) identificar los elementos que pueden hacer que una aseveración de conocimiento sea considerada confiable; 0 vi) valorar la contrastación de resultados entre pares o con otros grupos de trabajo.

En síntesis, a fin de orientar la enseñanza y evaluar la efectividad de la misma, cada trabajo ex perimental debe tener ex plíitos, objetivos de aprendizaje asociados con: i) lo conœeptual (conceptos, teoremas, representaciones, concebidos desde la teoría de CC), referidos al dominio metodológico y al dominio teórico, en función de la situación problemática; y ii) lo epistemológico.

A hora bien, considerando que el proceso de aprendizaje no es equivalente al proceso de producción de conocimientos en la ciencia (Kirschner, 1992) surge como inquietud: ¿cómo es el proceso de aprendizaje durante el desarrollo de un TL? ¿cómo facilitar el aprendizaje de los estudiantes en cada uno de los momentos del proceso de indagación que se lleva a cabo en el laboratorio? D ado que en este trabajo se concibe la actividad experimental como un espacio en el cual el estudiante se enfrenta a un problema que le demandará la resolución de un conjunto de tareas y subtareas que le son propias a la ciencia, las cuales son vistas como oportunidades para contribuir al desarrollo conceptual del estudiante, hemos considerado necesario comprender la actividad cognitiva subyacente al proceso de resolución de la situación del TL. En tal sentido, se ha elaborado un modelo que intenta interpretar el proceso de aprendizaje durante el desarrollo de un TL, con base a la teoría de Campos Conceptuales. 


\section{La dinámica del aprendizaje en el laboratorio desde la Teońa de Campos Conceptuales}

Si el Trabajo de Laboratorio consiste en una situación problemática cuya resolución demanda enfrentar diversos tipos de tareas, su abordaje resulta ser una actividad cognitiva compleja. Por ello, se propone un modelo interpretativo del desarrollo cognitivo de los estudiantes enfrentados a este tipo de situaciones experimentales, basado en la teoría de CC de Vergnaud (1990) con el complemento de Moreira e G reca (2002). El modelo propuesto, al que denominaremos: Modelo dinámico de Aprendizaje en los Trabajos de Laboratorio en Física (MATLaF), está representado en la Figura 2.

Como ya se dijo, el enfoque de laboratorio asumido, parte de la exposición de los estudiantes ante situaciones problema novedosas, aspecto central en la teoría de CC (Vergnaud, 1990) para que se produzca aprendizaje si se intenta la construcción significativa de esquemas científicos. Así, los estudiantes perciben la situación con sus esquemas y al no encontrar uno que se ajuste completamente, entran en una fase de reflexión y duda (ibidem); ante ésto, los estudiantes construyen MM que evolucionan hasta alcanzar su funcionalidad (Moreira, 2002). Estos modelos transitorios contienen elementos, del tipo invariantes operatorios que se mantienen y que unidos a otros IO del conocimiento del estudiante, permiten la identificación de la información pertinente al problema y la producción de predicciones e inferencias operativas para el TL, generando una solución tentativa. Durante la elaboración recursiva de los MM pueden intervenir factores externos, como: la interacción social con pares, la mediación del docente, la búsqueda de información desde otras fuentes, la realización de observaciones, los que incidirán progresivamente en precisar, modificar y enriquecer los invariantes operatorios. Se supone que esta actividad cognitiva inicial cesa una vez que los estudiantes se encuentran satisfechos con sus producciones y logran replantear el problema en términos de preguntas relevantes, es decir, preguntas acerca del mundo físico según variables pertinentes al problema a resolver (meta inicial). Así, se inicia la construcción progresiva de nuevos esquemas, o por lo menos una cierta estabilización de los MM, para la situación que permiten generar una primera secuencia de acciones. Resulta relevante hacer explícitos estos IO, para mediar en la construcción de los conocimientos de la ciencia a partir de ellos.

A partir de la reformulación de la situación y generación de las preguntas relevantes (primeras demandas del problema) y la primera secuencia de acciones, éstas surgen como nuevas subtareas: diseñar ex perimento, realizar mediciones, reoolectar y organizar datos, transformar y representar resultados, entre otras; todas típicas del quehacer del laboratorio en la ciencia. Cada subtarea se puede considerar como una situación-problema, donde para resolverla hay que construir una secuencia de acciones sobre la base de un esquema que puede o no estar en su estructura de conocimientos. En el último caso, la subtarea resulta ser novedosa, por lo que, su abordaje puede ser descrito como un proceso cognitivo similar al presentado en el parágrafo anterior.

Por otra parte, desde lo científico, las conceptualizaciones asociadas a los diferentes esquemas requeridos en el TL pueden referirse tanto al marco teórico-epistemológico como al marco metodológico, variando su énfasis de unas tareas a otras. Así, consideramos que en cada uno de los momentos identificados como propios del proceso de producción de conocimientos de la ciencia, y por ende, del trabajo de laboratorio centrado en situaciones-proble- 




\section{Figura 2}

Modelo dinámico de Aprendizaje en los Trabajos de Laboratorio en Física (MATLaF)

ma, ocurrirá un proceso de aprendizaje que puede ser comprendido y facilitado según el modelo MATLaF presentado. A tal fin, se ha derivado una metodología para el estudio de la efectividad de los TL en la enseñanza de la física orientados según el enfoque planteado, la cual se expone a continuación.

Propuesta metodológica para evaluar la efectividad de los trabajos de laboratorio orientados por el modelo de aprendizaje propuesto (MATLaF)

La investigación relativa al proceso de aprendizaje durante los TL según el enfoque asumido, puede orientarse atendiendo al modelo MATLaF. En este sentido, identificamos cuatro momentos importantes del proceso para la recolección de información con fines investigativos, los cuales son:

i) Identificar invariantes operatorios (I0) de los estudiantes asociados a la situación inicial. Partiendo del supuesto de que la situación problemática que se propone es novedosa para los estudiantes, se estima que éstos no tienen todas las competencias para abordarla de inmediato (Vergnaud, 1990), en consecuencia, tendrán que construir los modelos y esquemas necesarios 
para ello. Se propone entonces, una secuencia de actividades de diagnóstico de los razonamientos iniciales, que comienza con la presentación de la situación y la recolección por escrito de las respuestas individuales, complementadas con entrevistas (individuales o colectivas) a los estudiantes realizadas en distintos momentos durante el proceso de construcción de un esquema que les permita la formulación de las preguntas relevantes consensuadas y un primer plan de acción. Este diagnóstico permitirá inferir los IO iniciales. Los instrumentos para las recolección de datos propuestos son: situaciones con preguntas presentadas en forma escrita, guiones de preguntas para entrevistas.

ii) Identificar invariantes operatorios (I0) de los estudiantes, asociados con las subtareas propiamente ex perimentales. En la figura 1 se destaca en un recuadro las tareas-problema que están más relacionadas con el dominio metodológico que como se indicó, están interrelacionados con el dominio teórico. Dada la complejidad del mismo, por la variedad y cantidad de subtareas asociadas a las preguntas experimentales generadas, se analiza a cada una como una situaciónproblema que puede o no resultar novedosa para el estudiante, por lo que se suceden ciclos semejantes al anterior. Se propone entonces, el uso del registro anecdótico por parte de los estudiantes, en donde escriban todo lo que consideren necesario e importante de cada sesión de trabajo (reflexiones, decisiones, preguntas, predicciones, informaciones teóricas evocadas 0 extraídas de la búsqueda de información, procedimientos, datos, cálculos, otros,... ). Y como complemento, la observación y registro (escrito, audio o video) de las sesiones de trabajo por parte del investigador. El análisis de esta información permitiría obtener evidencias acerca de: a) los esquemas de asimilación utilizados por los estudiantes ante aquellas subtareas que resultaron conocidas; b) las subtareas que les resultan ser desconocidas a los estudiantes y los IO subyacentes al esquema reformulado o construido para abordarlas, y c) las representaciones simbólicas que producen en relación con los conceptos y la situación.

iii) E valuar aprendizajes logrados. El abordar una situación problema en el TL para lograr aprendizajes establecidos explícitamente (conceptual: teórico, metodológico y epistemológico), requiere tener evidencia de ellos. Esto es factible a partir de los reportes finales de los estudiantes, estrategia de comunicación típica en la ciencia. $\mathrm{O}$, mediante sus soluciones a otras situaciones de la misma clase que pudieran ser presentadas después del TL. Ambas vías son una forma de establecer la efectividad del TL, en términos del cambio entre estado inicial y estado final.

Se propone que cada trabajo de laboratorio culmine con la comunicación a los pares, oral y/ o escrita. El análisis y reflexión acerca de esta presentación, en función de los elementos del quehacer de la ciencia (figura 1) y de las relaciones establecidas entre ellos, permitirá inferir el aprendizaje de orden epistemológico generado con el TL.

iv) Comparar ex pectativas de los estudiantes (y del docente)on logros obtenidos. Existe consenso en la importancia de tomar conciencia de los aprendizajes que se esperan alcanzar, reflexionar acerca de los logros y de las posibles discrepancias. En tal sentido, el modelo MATLaF permite incluir momentos de reflexión individual: a) antes de iniciar las tareas metodológicas; y b) después de la presentación final del TL.

En cada situación de trabajo de laboratorio se ponen en evidencia algunos aspectos relevantes del quehacer científico (Andrés e Pesa, 2003). Si en este espacio se promueve la reflexión epistemológica de manera intencional, se estará favoreciendo la construcción de una visión acerca de la ciencia en los estudiantes. Así, en el marco del desarrollo de los TL bajo el 
enfoque propuesto, resulta relevante evaluar la efectividad que tienen en cuanto a la evolución de las concepciones epistemológicas de los estudiantes, mediante su evaluación antes y después de un ciclo de TLs. Para ello se sugiere el cuestionario CAEF (ibidem), elaborado para explorar las ideas de los estudiantes acerca de su visión respecto de la actividad experimental en la física.

\section{Consideraciones finales}

El modelo MATLaF es una herramienta que consideramos pertinente para la comprensión y estudio del aprendizaje durante el TL centrado en la resolución de situaciones problema. En tal sentido, se propone identificar las demandas conceptuales propias del laboratorio según los contenidos desde la física y caracterizar las tareas del laboratorio en función de la complejidad conceptual para los estudiantes frente a TL en cursos de física, con diferentes contextos teóricos y diferentes niveles educativos.

Por otra parte, al considerar el aprendizaje en el laboratorio como un espacio de desarrollo conceptual, donde ocurre la aplicación de conocimientos teóricos y metodológicos para resolver situaciones que tienen soluciones experimentales, resulta necesario identificar los IO utilizados por los estudiantes en situaciones semejantes -los cuales pueden diferir respecto de lo acordado en la ciencia- para así intentar ponerlos en evidencia y construir con ellos las conceptualizaciones de ciencia.

A partir del modelo también se pueden derivar orientaciones didácticas tanto para el docente como para los estudiantes para la realización de TL abordados a partir de situaciones. Ello implica hacer investigaciones de campo sobre las propuestas didácticas derivadas del MATLaF para la enseñanza de la física en el laboratorio.

Como plantea Vergnaud (1990), un campo conceptual presenta dos caras de una misma moneda, incluye un conjunto de reglas y prooedimientos para la aplicación de un conjunto de conoptos y relaciones (representados a diferentes niveles) en la solución de una variedad de situaciones pertinentes. En consecuencia, dado que en los TL predomina el aprendizaje de nuevas conceptualizaciones propias del campo metodológico, es pertinente definir el campo conceptual asociado a esta actividad dentro de la física, estableciendo las dases de situaciones, y los conœeptos y relaciones asociados a ellas que resultan ser característicos de la actividad experimental en la enseñanza de la física.

En síntesis, consideramos que: el modelo MATLaF (figura 2) es un aporte teórico con gran potencial para comprender y facilitar el proceso de aprendizaje durante la resolución de las tareas y subtareas propias de los $\mathrm{TL}$, de lo cual se tienen ya algunas evidencias empíricas (Andrés, 2005; Andrés et al., 2004; 2006) Además, el plan general para guiar el trabajo de laboratorio a partir de situaciones problemáticas, derivado desde la ciencia, al representarlo en la $\mathrm{V}$ epistemológica (figura 1), permite a los estudiantes tomar conciencia de la interrelación teoría-experimento. Por lo tanto, concluimos que estas dos perspectivas, la cognitiva y la epistemológica, constituyen un referencial fructífero para la investigación acerca del aprendizaje del domino metodológico en los trabajos de laboratorio en la enseñanza de la física, y en consecuencia, derivar sugerencias didácticas para el desarrollo de los TL, con mayor eficiencia y efectividad. 


\section{Referências}

AND RÉS, M. M. La formación del docente de física: realidad y perspectivas. Trabajo de ascenso a Profesor Titular, Univ. Pedagógica Experimental Libertador-IPC. Caracas. Venezuela, 2002.

Diseño del trabajo de laboratorio con bases epistemológicas y didácticas: caso carrera de docentes de Física. España. 2005. Disertación (D octoral) - Universidad de Burgos.

AND RÉS, M. M.; PESA, M. Criterios para la evaluación de las concepciones de estudiantes de profesorado de Física acerca de la actividad experimental en la Ciencia. In: ENCUENTRO IBEROAMERICANO SOBRE INVESTIGACIÓN EN EDUCACIÓN EN CIENCIAS, 1., Actas, Universidad de Burgos, Burgos p. 443-458, 2003.

. Conceptos-en-acción y Teoremas-en-acción en un Trabajo de Laboratorio de Física. Revista Brasilera Pesquisa Em Educacao Ciencias. Brasil, v. 4, n. 1, p. 59-75, ene/ abril 2004.

AND RÉS, M. M.; PESA, M.; MENESES, J. D esarrollo conceptual acerca de ondas mecánicas en un laboratorio guiado por el modelo MATLaF. Revista Electrónica de Enseñanza de las Ciencias, v. 5, n. 2, p. 280-288. D isponível em: <http:/ / www.saum.uvigo.es/ reec/ >D OAJ y Ulrich, junio, 2006.

BARBERÁ, O.; VALDÉS, P. El trabajo práctico en al enseñanza de las ciencias: una revisión. Enseñanza de las Ciencias, v. 14, n. 3, p. 365-379, 1994.

BRAVO, S.; PESA M. Propagación de pulsos. Una interpretación del razonamiento de los estudiantes. In: SIMPO SIO NACIO NAL DE INVESTIGAD ORES EN ED UCACIÓN EN FÍSICA, 7., Argentina, 2004. Memorias... La Pampa, Argentina, 2004a.

. La "ola en el estadio": ¿Movimiento ondulatorio? Una interpretación del razonamiento de los estudiantes. In: SIMPOSIO NACIONAL DE INVESTIGAD ORES EN EDUCACIÓ N EN FÍSICA, 7., Argentina, 2004. Memorias... La Pampa, Argentina, 2004b.

ESCUDERO, C.; MOREIRA, M. A.; CABALLERO, C. Teoremas-en-acción y Conceptos-enacción en clases de física introductoria de secundaria. Revista electrónica de Enseñanza de las Ciencias, v. 2, n. 3, 2003. Disponível em: <http:/ / www.saum.uvigo.es/ reec> .

DRIVER, R.; LEACH, J.; MILLAR, R.; SCOTT, P. Young people's images of Science. Buckingham: Open University Press, 1996.

DUIT, R. The construvtivist views: a fashionable and fruitful paradigm for Science Education. research and practice. In: STEFFE, L.; GALE. Constructivism in E ducation. Lawrence Erlbaum Associates. Inc N.J., 1995. p. 271-286.

DUSCHL, R. Making the nature of science explicit. In: MILLAR, R.; LEACH, J.; OSBORNE, J. Improving science education. Philadelphia: O pen University Press, 2000. 
GIL, D.; CARRASCOSA, J.; FURIÓ, C.; MTNEZ-TORREG OSA, J. La Enseñanza de las Ciencias en la Educación Secundaria. Cuadernos de Educación, v. 5. Horsorí, España, cap. III, 1991.

G IL, D.; VALD ES, P. La orientación de las prácticas de laboratorio como investigación: un ejemplo ilustrativo. Enseñanza de las Ciencias, v. 14, n. 2, p. 155-164, 1996.

HOD SO N, D. Hacia un enfoque más crítico del trabajo de laboratorio. Enseñanza de las Ciencias, v. 12, n. 3, p. 299-313, 1994.

KIRSCHNER, P. A. Epistemology, practical work and academic skills in science education. Science and Education, v. 1, p. 273-299, 1992.

LEACH, J. Learning science in the laboratory: the importance of epistemological understanding. In: LEACH, J.; PAULSEN, A. (ed.) Practical work in Science Education. Kluwe Academic Pubs, 1999. p. 134-147.

LEACH, J.; MILLAR, R.; RYDER, J.; SÉRÉ, Ma-G.; HAMMELEV, D.; NIED DERER, H.; TSELFE S, V. Survey 2: students' images of science as they relate to labwork learning. Labwork in science education. Working paper 4, 2001. Disponível em: <http:/ / www.cordis.lu/ tser/ src/ ct2001w.htm>. Acesso em: 2002.

MOREIRA, M. A. La teoria de lo campos conceptuales de Vergnaud, la enseñanza de las ciencias y la investigación en el área. Traducción de Isabel Iglesias. Investigações em Ensino de Ciências, v. 7, n. 1, art. 1, 2002. D isponível em: <http:/ / www.if.ufrgs.br/ ienci> Acesso em: 2003.

MOREIRA, M. A.; GRECCA, I. M. Além da detecção de modelos mentais dos estudantes, uma proposta representacional integradora. Investigações em Ensino de Ciências, v. 7, n. 1, art. 2, 2002. Disponível em: <http:/ / www.if.ufrgs.br/ ienci>.

NOVAK, J.; GOWIN, D. B. Learning how to learn. Cambridge: University Press, 1984.

ROD RÍG UEZ P., M.; MOREIRA, M. A. La teoría de los campos conceptuales de Vergnaud. In: MOREIRA, M. A. (Org.) La teoría de los campos conceptuales de Vergnaud, la enseñanza de las ciencias y la investigación en el área. Brasil: Instituto de Física, Universidad Federal de Rio Grande do Sul, 2004.

SALINAS, J. Las prácticas de Física Básica en laboratorios universitarios. España. 1994. Tésis (D octoral) - Universidad de Valencia.

SÉRE, Ma-G. Towards renewed research questions from the outcomes of the european project labwork in Science Education. Science Education, p. 625 -644, 2002.

STIPCICH, S.; MOREIRA, M. A.; CABALLERO, C. Una interpretación de las opiniones de ingresantes a la universidad sobre la noción de interacción. Revista Electrónica de Enseñanza de las Ciencias, v. 3, n. 1, 2004. D isponível em: <http:/ / www.saum.uvigo.es/ reec>. 
TO BIN, K.; TIPPINS, D.; G ALLARD, A. J. Research on instrucctional strategias for teaching Science. In: GABE L, D. (ed.). Handbook of Research on Acience Teaching and Learning. New York: NSTA, 1994.

VERG NAUD, G. A classification of cognitive tasks and operations of thought involved in addition and substraction problems. In: CARPENTER, T.; MO SER, J.; RO MBERG, T. Addition and substraction: a cognitive perspective. New Jersey: Lawrence Erlbaum, 1982.

. La théorie des champs conceptuels. Traducido por Juan Godino. Recherches en Didáctique des Mathématiques, v. 10 , n. 2, p. 133-170, 1990.

. Multiplicative conceptual field: what and why? In: GUERSHO N, H.; CONFREY, J. (Eds.). The development of multiplicative reasoning in the learning of mathematics. New York: State University of New York Press, 1994.

. Education the best portion of Piaget's heritage. Swiss Journal of Physics, v. 55, n. 2/ 3, p. 112-118, 1996.

. A comprehensive theory of representation for mathematics education. Journal of Mathematical Behavior, v. 17, n. 2, p. 167-181, 1998.

WEIL-BARAIS, A.; VERGNAUD, G. Students conception in Physics and Mathematics: biases and helps. In: CAVERNI, J. P.; FABRE, J. M.; G O NZÁLEZ, M. (Eds.). Cognitive biases. North Holland: Elsevier Science Publishers, 1990. p. 69-84. 